\title{
SLR and GNSS Test Field for Global Geodetic Network Assessment in Riga
}

\author{
Janis Kaminskis (iD ${ }^{*}$, Lubova Sulakova ${ }^{2}$, Kalvis Salmins (iD ${ }^{3}$, \\ Janis Kaulins (iD ${ }^{4}$, Lauris Goldbergs ${ }^{5}$ \\ 1, 2, 5 Geomatics Department, Faculty of Civil Engineering, Riga Technical University, Riga, Latvia \\ ${ }^{3,4}{ }^{4}$ Institute of Astronomy, Latvia University, Riga, Latvia
}

Received 28 February 2020; accepted 31 March 2020

\begin{abstract}
The basic aim is to contribute to the world geodetic space in line with today's scientific achievements. Riga geodynamic site is a thankful place for this, as it has long-term SLR observations and the longest GNSS records in Latvia. The goal is establishment of regional long-term geodetic monitoring station at LU Institute of Astronomy, Riga, Kandavas street 2, by joining at least two space geodetic technologies - the already installed laser-telescope LS-105 and GNSS - collocated, but not sufficiently linked. The capability of geodetic GNSS observations would uniquely complement Riga GNSS station and allow to determine more accurate coordinates of the LS-105 laser telescope and the long-term changes needed to accurately measure the positions of Earth satellites and other similar space objects. GNSS Observation Station will contribute to the development of positioning and position long-term change to accuracy of less than 1mm, one of the current global goals of GGOS. We plan to solve the problem with the exact position of the telescope LS-105 it will contribute to the development of scientific research and applied potential of the LU Satellite Laser Ranging station. From the national point of view geodetic station serves as an important point for Latvian National Geodetic Network, long term large infrastructure planning, engineering communications, cartography, etc. From a global perspective the station will be one of very few such stations in the region and the only one in the Baltics capable of valuable contribution to ITRF network. Preparatory work for the study has started by selecting and consolidating geodetic points for further measurements.
\end{abstract}

Keywords: geodesy, satellite laser ranging, laser telescope, data processing, collocated techniques.

\section{Introduction}

One of the divisions of higher geodesy is celestial geodesy or space geodesy. This requires observing and estimation techniques to use accurate measurements on, from, or between Earth satellites or other extraterrestrial bodies to solve geodesic problems. Key issues addressed by cosmic geodesy are: accurate definition of global, regional and local threedimensional positions; parameter determination of the Earth's gravity field (accurate geoid); measurement and modeling of geodynamic phenomena (pole movement, earth rotation, earth's crust deformation) (Clark, 2003). The satellites of the earth play a major role in geodesy. These can be used as targets for high altitude that can be seen from long distances. Satellites can be described as fixed control points in a global three-dimensional network, according to trigonometric networks. When satellites are viewed from several stations concurrently, the positions of their connection vectors are collected, and it is meaningless that gravity determines the trajectory of the satellites. Many vectors like this form a global vector network-cosmic triangulation. The main advantage of satellite tracking relative to traditional geodesy is long distance measurements, which made it possible to create geodesic relations between continents and islands. The Moon was observed earlier, then artificial satellites, using this feature. Optical techniques have captured pictures of starlit or blinking satellites in the background using strong lenses. The first experiments were to chart satellite orbits and establish the Earth's mathematical structure. Around 60's and 70's this method of calculation dominated (Gross, 2020). One of the last spacecrafts detected by optical approach (launched in 1986) was the Japanese AJISAI satellite (Bikše et al., 1992). The classification of observation techniques may also be based on the relationship between the observer and the target. The following techniques could be broken down into Earth/Space Methods directional measurements from photos; satellite laser positioning, doppler positioning, global Navigation Satellite Systems. Space/Earth Methods - radio altimeter; laser altimeters; satellite gradiometry. Space/Space - Satellite/

${ }^{*}$ Corresponding author. E-mail: janis.kaminskis@rtu.lv

\section{Copyright (C) 2020 The Author(s). Published by VGTU Press}

This is an Open Access article distributed under the terms of the Creative Commons Attribution License (http://creativecommons.org/licenses/by/4.0/), which permits unrestricted use, distribution, and reproduction in any medium, provided the original author and source are credited. 
satellite tracking (Gross, 2020). Figure 1 space geodesy techniques (IAG web site, 2020, https://www.iagaig.org/topic/3).

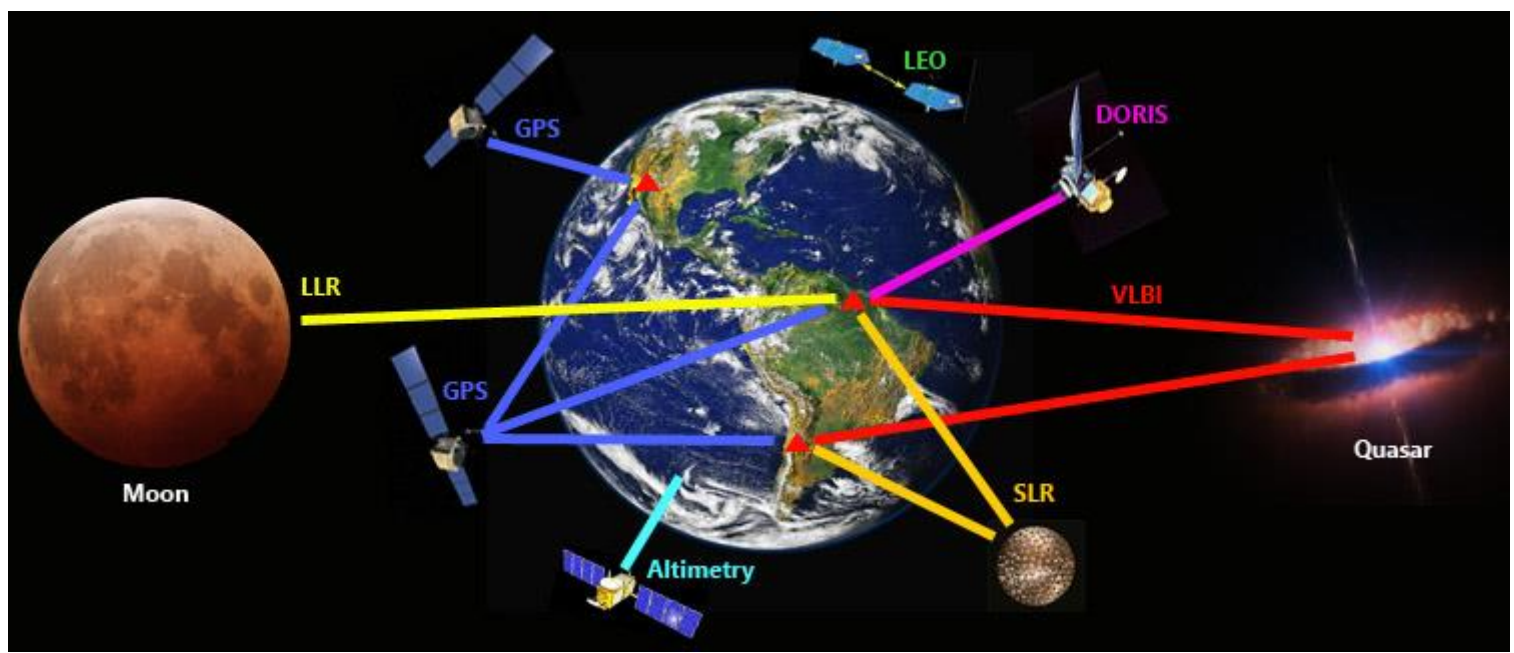

Figure 1. Space geodesy techniques (https://www.iag-aig.org/topic/3)

Space geodetic technologies are also used for geodetic control, establishing geodetic connections between islands, continents, mountains and other areas not accessible by traditional geodetic techniques, observing, developing and managing geodetic networks. In functional geodesy, GPS measurements are widely used, both in field measurements and it's technology power in navigation and marine geodesy; space surveying also plays an essential role in, for example, the accurate navigation of land, sea and air vehicles, mapping and exploration, hydrography and oceanography. The acceleration, rotation, waves and crustal motions of Earth's poles are studied in geodynamics via interstellar geodesy (Seeber, 2003). Cosmic geodesy has led to the development of a number of specific areas closely related to it, such as the analysis of ice motions in glaciology, atmospheric tomography and the identification of satellite orbits. The use of cosmic geodesy is increasing. They are influenced by the rapid development of this area, the everincreasing number of satellites in space, the improvement in the accuracy that can be achieved, the more sophisticated, more compact, user-friendly devices (Gross, 2020).

Space geodesy methods enables various scientific organizations to provide wide range of scientific disciplines, including seismology, geodynamics, climate science, hydrology, oceanography, meteorology, and space weather assumptions (International Laser Ranging Service, 2019). Satellite Laser Ranging (SLR) and Lunar Laser Ranging (LLR) - in this technique, a laser signal is transmitted from a ground-based station, reflects off specially designed mirrors (retro-reflectors) placed on artificial satellites (SLR) or on the moon (LLR), and is received back at the station. Satellites and the moon orbit the Earth due to the Earth's gravitational pull. This technique therefore provides a unique connection between the Earth's surface and its gravity field, which reflects the distribution of mass deep within the planet. Global Navigational Satellite Systems (GNSS) this is a generic term for satellite navigation systems that provide autonomous spatial positioning with global coverage. The U.S. NAVSTAR Global Positioning System (GPS) falls into this category; other countries also have or are developing GNSS systems. Coded signals transmitted from multiple satellites and received by multiple GNSS receivers allow time delay, and indirectly distance, measurements between the satellites and GNSS receivers (Jokela et al., 2006).

\section{Satellite laser ranging}

Satellite laser ranging (SLR) is also one of the space geodetic observation techniques. A global station network armed with SLR can instantaneously direct an ultrashort light pulses to satellites equipped with special reflectors. It offers instantaneous millimeter level resolution measurements that can be collected to provide precise orbits and a variety of important scientific items. Basically, the time of the laser pulse travelling between a ground station and a satellite is key component and mainly observed in laser distance measurement of satellites. In the ground station, a short laser pulse is generated and transmitted to the satellite through an optical system. To Begin a digital time interval monitor (user clock) a portion of the outgoing laser pulse is used as a starting sing. The target satellite has suitable retroreflectors that reflects laser beam and the mirrored pulse is detected back at the base station on earth than amplified \& analyzed. The signal time is extracted from the two user clock readings and is scaled to the distance $-\mathrm{d}$, with the signal velocity c. The formula of basic observation is therefore very simple:

$$
d=(\Delta t / 2) \times c .
$$


In the United States in 1961, the use of a laser pulse system for Earth observation satellites began to develop. The Beacon Explorer-B was the first satellite fitted with retroreflectors and launched in 1964. In 1965, the first useful reflections were obtained and given several meters of accuracy. Progress was very rapid in the years that followed, and measuring accuracy improved from a few meters to a few millimeters. SLR stations around the world have been deployed. There are currently 45 base stations ILRS (International Laser Ranging Service) station network. Measurements are also carried out by many independent observers at various observatories (Schuh \& Behrend, 2012). The laser systems are divided into several groupings based on conception and achievements. So called first-generation laser devices operated in the range 1-6 m, with pulse lengths from 10 to $40 \mathrm{~ns}$, armed with ruby lasers. Laser pulse length in the second generation was between 2 and $5 \mathrm{~ns}$, advanced methods for the measurement with precision upwards from 30 to $100 \mathrm{~cm}$. Laser systems of third generation use laser pulses from 0.1 to $0.2 \mathrm{~ns}$. Newest generation of lasers can pump out even shorter pulses, with repeatable frequency of $2 \mathrm{kHz}$ (2000 impulses in second) that's about 10 picoseconds. First- and second-generation devices are essentially unused for scientific observation nowadays. The main benefits of SLR are: very high precision (mainly due to the advantageous properties of lights absorption), stability of satellites designed for direct observation by SLR and not fitted with active components, confirmation of geocentric coordinates, can be used alone without other space geodesy techniques, optional for active orbital navigation systems such as PRARE, DORIS, GPS ; accuracy is not affected by ionosphere (National Academies of Sciences, Engineering, and Medicine, 2020). And key disadvantages: Climate dependency (clouds, rain etc.); high cost of station operation; inhomogeneous distribution of information relative to GPS, DORIS or VLBI.

Precise laser positioning is usually performed on satellites equipped with special reflectors (can be done without special reflectors but decrease in accuracy will be shown). In most cases, to achieve the required energy level, some individual reflectors with a diameter of 2 to $4 \mathrm{~cm}$ are assembled in certain blocks one certain satellites. Since reflectors are passive instruments, they can be mounted very simply; therefore, there is a very high number of satellites armed with retroreflectors. In special satellite missions, such as altimeter satellites or gravity field observation missions, the SLR technique is used to provide accurate orbit data in general most satellites with a scientific need for precise orbits are fitted with retroreflector blocks that function as back-up systems. There are also satellites in unique orbits with only soul purpose to provide a target for SLR. These satellites were built with optimum geometry and specific orbital characteristics. These spacecrafts, for example, AJISAI, STARLETTE, ETALON-1/2, STELLA, LAGEOS-1/2, are equipped with retro-reflector modules only. They all have a spherical shape, and the reflectors are all over the surface and attached to the satellite's center of gravity. Such a spacecraft is used primarily to solve the problems of geodynamics (Kempf, 2008).

This method has proved to have a very good accuracy potential from the very first observations of the SLR and will be widely used in geodesy and geodynamics. New applications are emerging as accuracy improves over time. The SLR received a precision of about $3 \mathrm{~m}$ in 1965, and then the main object of study was the gravitational field. Accuracy reached $10 \mathrm{~cm}$ in the eighties, enabling the study of Earth's tides and rotation. Shortly thereafter, accuracy was only a few centimeters, and tectonics were already being studied on the Earth's plates. In the mid-nineties, when accuracy was less than a centimeter, the deformation of the Earth's crust could be recorded in certain regions and accurate Earth orientation data could be obtained (Gross, 2020).

\section{Riga astro-geodynamic observatory}

The term astro-geodynamic observatory refers to spatial observation stations using more than one form of observation and in this case Riga astro-geodynamic observatory matches needed conditions. Methods for monitoring SLR and GPS are co-located in Riga.

In addition to astronomical observations, Riga Station also conducts gravimetric and groundwater level measurements. Figure 2 Riga's station (http://www.igs.org/images/site/riga002.jpg). The main GNSS receiver which is longest running receiver in Baltic states was installed in the Observatory in 1995 and included in the 1996 European GNSS Standalone Station Support Network (EUREF) and has also been the base station for the IGS network since 2005. Riga Station is the Baltic States first IGS station. The inclusion of IGS in the EUREF network involved several years of regular Riga station measurements. The benefit was also to help maintain coordinate systems by an established satellite laser positioning station nearby. The station is currently operating the Leica AR25 receiver after the replacement of last LEICA GRX1200PRO and other various GPS receivers. The new AR25 has been designed for all existing and currently planned signals of the GPS, GLONASS, Galileo, BeiDou, QZSS and NavIC systems. The GPS antenna is mounted on the roof of the clock cellar in a stable position which is essentially a 5-meter long concrete cylinder fixed to dolomite bedrock 12 meters deep. The antenna itself is mounted on a concrete posted metal tripod. The antenna's location on the post is determined by a geodesic mark under the antenna. 


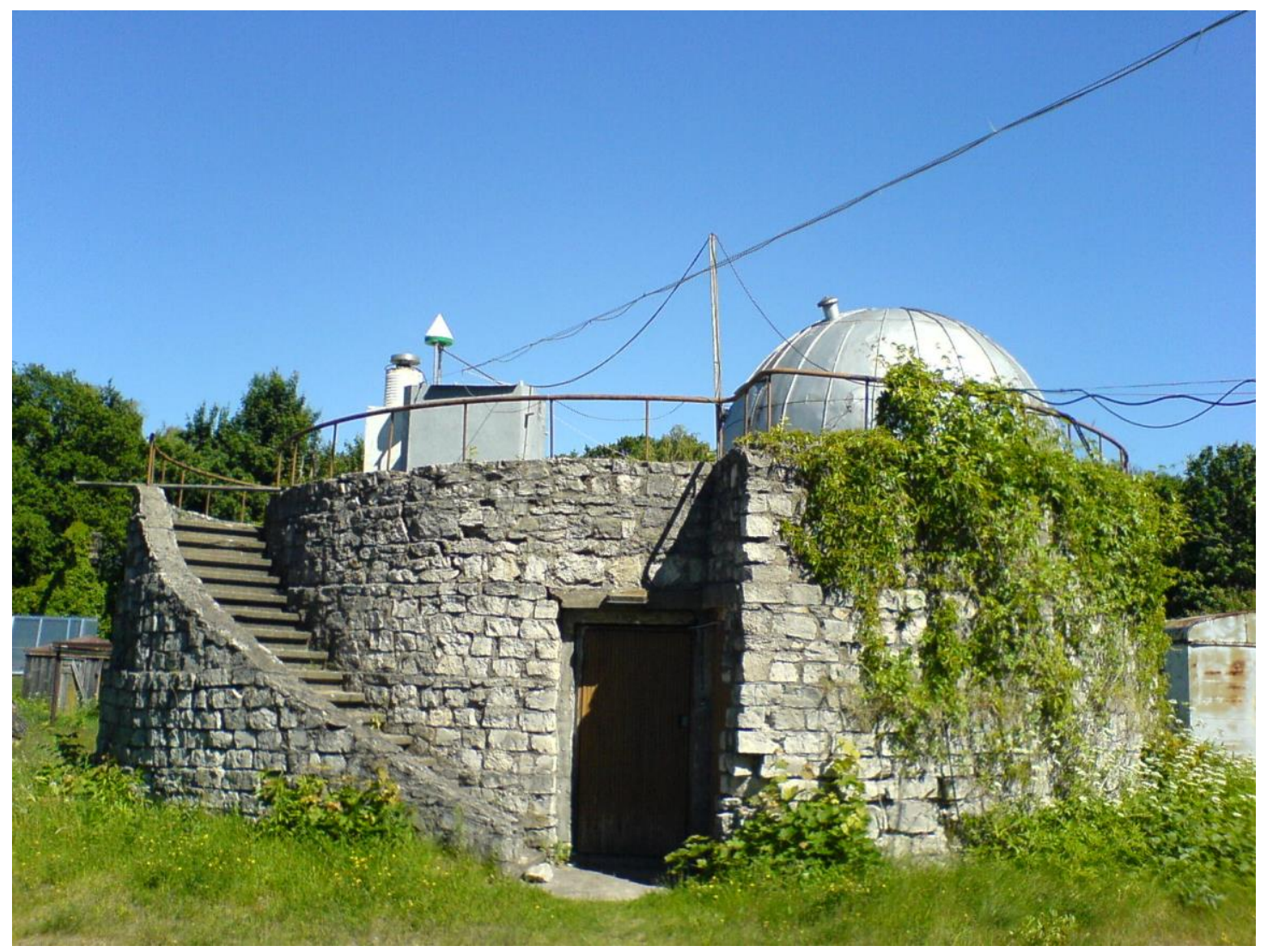

Figure 2. Riga's station (http://www.igs.org/images/site/riga002.jpg)

The LS-105 telescope, produced in 1985, is used. A 1-metre-wide telescope mirror was also preserved, and its reflective surface was restored in 2007. A concept of "three to one" was developed for the new laser rangefinders LS-105. The three major functions of the rangefinder are done using same the main optical system - the collimation and a deviation of a beam, the receiver of the reflected pulse and a guiding and aiming. The central optical unit consists of a $100 \mathrm{~cm}$ diameter dish with $11.6 \mathrm{~m}$ focal point. A telescope mount is a classic stepper-azimuth motor which can be precisely moved by software. The worm gear is powered by Azimuth engines. The height of the worm gear is four times the height of the azimuth because it must travel just 90 degrees instead of 360 degrees. Such a system provides the telescope with steady, smooth movement at a total $2^{0} 55$ per second speed. You can also manually adjust the position of the telescope using the limbs of azimuth and height (IDS web site, 2019).

\subsection{Site description}

Reference point survey (local tie) is performed in Riga, Latvia, not far from the city center or old town of Riga. Riga, Latvia's capital, is located on the coast of the Baltic Sea at the mouth of the Daugava River. Riga is the Baltic states largest city. Riga is located at $56^{\circ} 58^{\prime}$ North and $24^{\circ} 8^{\prime}$. More precise the site is located at the University of Latvia botanical garden where astronomical observatory has taken its place since 1957 (IVS web site, 2019). Observatory is managed by Institute of Astronomy that performs research and development for special data structures.

From a geodetic point of view, the observatory is equipped with several scientific instruments:

- Longest running GNSS receiver in Baltics;

- One of high precision SLR stations in the world.

Co-located points:

- Recently installed monuments (in total - three);

- GNSS receiver reference point right below the antenna;

- SLR reference point (axis intersection point);

-SLR reference point (outside the actual laser telescope).

Other points of interest:

- Geodetic mark on a concrete block;

- Two broadhead nails imbedded into asphalt. 


\subsection{Survey conditions}

Local tie survey was conducted 21th of November year 2019, for late autumn day the weather was favorable for such measurements, it was cloudy with gentle breeze of wind, temperature right about $12{ }^{\circ} \mathrm{C}$, which prevented the air from any distorting effects. Atmospheric pressure readying $755 \mathrm{mmHg}$, humidity around $85 \%$. This kind of values was set into total station for all local tie measurement process.

Used instruments and accessories:

- Topcon OS-103 total station (3", precision);

- 3 Topcon Hyper SR GNSS receivers;

- mini prisms;

- tribraches and carriers. Specification of Total station:

- EDM distance standard deviation of a single measurement: $2 \mathrm{~mm}+2 \mathrm{ppm}$;

- Minimal resolution/accuracy: 1"'/3"'(Dolan, 2013);

- Instrument calibrated by the SIA GeoStar. Auxiliary Equipment:

- Meteoprog.lv: Temperature, atmospheric pressure;

- human senses: wind speed, cloud coverage.

(Can't talk about absolute accuracy in these variables due to methods of gathering information).

\subsection{Observations GNSS point RIGA00LVA}

The main antenna was not removed from its station during this survey to allow the continuation of the data and to avoid the risk of the antenna not being re-centered in the same position which could cause a possible shift in the time series. An indirect survey of the GNSS antenna reference point was conducted from each of the pillars used in this survey. To estimate the horizontal position of the antenna sets of angular observations were made to specific symmetrically coupled points on the external profile of the antenna a hexagon bolt slightly above reference point. By making use of the symmetrical properties of the object it was possible to intersect the observations and obtain direction observations to the central axis. Using triangulation, the horizontal position of the ARP was estimated. To estimate the height of the reference, point a simple levelling connection was made to measured point. Using the height difference acquired between the antenna reference point and the anchor bolt the ARP was derived (0.045 m) (Gross, 2020).

\subsection{Observations Terrestrial network survey}

As precise as possible EDM traverse was conducted between all 3 ground control poles and other seeable points of interest. Observations were completed in both faces and both directions if possible Horizontal angles, slope distances and zenith distances were recorded. Atmospherics were recorded at the start of the surveying process. Precise levelling wasn't conducted this time. For attaching to Latvia's height system, a precise levelling results were used from 2011, and precisely levelled points were surveyed using total station. Precise leveling was carried from first class leveling point " 1297 " that still stands and trough some second-class leveling points that in nowadays aren't available. For precise leveling precision digital level Trimble DiNi12 were used, with precision $0.3 \mathrm{~mm}$ on every kilometer. Leveling from point 1297 were divided in two parts.39 Precise leveled height to a known point within the surveying site will be used to attach local tie to Latvia's Height system. When adjusting total station measurements only vertical values will be used from these know points as they aren't the focus of this surveying task. Total station survey will be divided into 3 parts as all the new monuments must be considered and every possible/seeable measurement was attempted. All points and additional points of interest that can increase precision or are deemed as highly stable was measured as well (Gross, 2020).

\section{Conclusions}

The history of both GNSS measurements and SLR observations accumulated at Riga station has been around for decades. Already in the nineties the first geodetic interconnection of these different observations was made, or local geodetic network was started. However, at that time we did not have the exact geodetic tools that we have today. This includes the use of a newer and more accurate time standard developed and manufactured in Riga for SLR observations. The telescope's optical system has been restored and the SLR has been upgraded to its capabilities. We also have the latest and most accurate Leica Nova TS60 tachometer angular accuracy of 0.5 " (sub-second) and distance accuracy of $0.6 \mathrm{~mm}+1 \mathrm{ppm}$ (sub-millimetre). Aware of these facts, we aim to provide spatial geodetic coupling and temporal accuracy with sub-millimeter accuracy. 
Our planned contribution to the maintenance of the global coordinate space is crucial to the growth or increase of the role of the SLR, in line with international research recommendations. By providing a global geodetic reference system with accuracy of $1 \mathrm{~mm}$, we can analyze and investigate not only geodynamic processes, but also follow environmental processes, sea level changes, ecological aspects, long-term planetary changes, providing a reference system for Interferometry Synthetic Aperture Radar (InSAR) and its applications development. InSAR today is a relatively fashionable thing, with new discoveries and innovative applications expected. Realization requires an accurate global reference system, which can only be achieved through the successful integration of connected geodetic observation systems or using collocated space geodetic techniques.

\section{Disclosure statement}

Authors declare that we don't have any competing financial, professional, or personal interests from other parties.

\section{References}

Bikše, J., Lapuška, K., \& Lazdāns, J. (1992). Latvijas trigonometriskā tīkla piesaiste pasaules geodēziskajai sistēmai. Riga Technical university geomatics institute, Riga.

Clark, B. (2003). A review of the history of VLBI. In Radio Astronomy at the Fringe ASP Conference Series (Vol. 300, pp. 1-8).

Dolan, T. (2013). Arequipa ITRF co-location survey service. http://itrf.ensg.ign.fr/doc_ITRF/RT186_V1_DONAL_ArequipaITRF2013.pdf

Gross, R. (2020). Space geodesy reference points and stability. Riga Technical University, Riga.

IAG web site. (2020). Description of the global geodetic reference frame. https://www.iag-aig.org/topic/3

IDS web site. (2019). International DORIS service frame. https://ids-doris.org/

IGS web site. (2020). International GNSS service. http://www.igs.org/images/site/riga002.jpg

International Laser Ranging Service. (2019). Monthly Report Cards. SLR Global Performance Report Card: November 1, 2018 through October 31, 2019.

https://ilrs.cddis.eosdis.nasa.gov/network/system_performance/global_report_cards/monthly/perf_201910_wLLR.html

IVS web site. (2019). International VLBI service for Geodesy \& Astrometry frame. http://ivscc.bkg.bund.de/about/vlbi/whatis.html

Jokela, J., Hakli, P., Uusitalo, J., Piironen, J., \& Poutanen, M. (2006). Control measurements between the geodetic observation sites at Metsähovi. In H. Drewes (Ed.), Geodetic reference frames. Springer. https://books.google.lv/books?id=qs16DBf1arYC\&printsec=frontcover\&dq=control+measurements+between+the+geodetic +observation+sites \&hl=lv\&sa=X\&ved=0ahUKEwi837rF8OTmAhUSSxUIHaW7CikQ6AEIKDAA\#v=onepage \&q=control $\% 20$ measurements $\% 2 \% 200$ between $\% 20$ the $\% 20$ geodetic $\% 20$ observation $\% 20$ sites $\& \mathrm{f}=$ false

Kempf, Y. (2008). The use of VLBI as an investigation tool in geodesy. Introduction to Geophysics.

National Academies of Sciences, Engineering, and Medicine. (2020). Evolving the geodetic infrastructure to meet new scientific needs. The National Academies Press. https://doi.org/10.17226/25579

Schuh, H., \& Behrend, D. (2012). VLBI: A fascinating technique for geodesy and astrometry. Journal of Geodynamics, 61, 68-80. https://doi.org/10.1016/j.jog.2012.07.007

Seeber, G. (2003). Satellite geodesy ( $2^{\text {nd }}$ completely revised and extended edition). Walter de Gruyter. https://doi.org/10.1515/9783110200089 